\title{
Variations in the Quantum Efficiency of Multiple Exciton Generation for a Series of Chemically Treated PbSe Nanocrystal Films
}

\author{
Matthew C. Beard, ${ }^{\star, t, \downarrow}$ Aaron G. Midgett, ${ }^{\dagger, \S}$ Matt Law, ${ }^{\dagger, \neq, l}$ Octavi E. Semonin, ${ }^{\perp}$ \\ Randy J. Ellingson, ${ }^{\#}$ and Arthur J. Nozik ${ }^{\star, \neq, \S}$ \\ Basic Sciences Center, National Renewable Energy Laboratory, Golden, Colorado \\ 80401, Department of Chemistry and Biochemistry and Department of Physics, \\ University of Colorado, Boulder, Colorado 80309
}

Received November 26, 2008; Revised Manuscript Received January 8, 2009

\begin{abstract}
We study multiple exciton generation (MEG) in two series of chemically treated PbSe nanocrystal (NC) films. We find that the average number of excitons produced per absorbed photon varies between 1.0 and $2.4( \pm 0.2)$ at a photon energy of $\sim 4 E_{g}$ for films consisting of $3.7 \mathrm{~nm}$ NCs and between 1.1 and $1.6( \pm 0.1)$ at $h v \sim 5 E_{g}$ for films consisting of $7.4 \mathrm{~nm} \mathrm{NCs}$. The variations in MEG depend upon the chemical treatment used to electronically couple the NCs in each film. The single and multiexciton lifetimes also change with the chemical treatment: biexciton lifetimes increase with stronger inter-NC electronic coupling and exciton delocalization, while single exciton lifetimes decrease after most treatments relative to the same NCs in solution. Single exciton lifetimes are particularly affected by surface treatments that dope the films n-type, which we tentatively attribute to an Auger recombination process between a single exciton and an electron produced by ionization of the dopant donor. These results imply that a better understanding of the effects of surface chemistry on film doping, NC carrier dynamics, and inter-NC interactions is necessary to build solar energy conversion devices that can harvest the multiple carriers produced by MEG. Our results show that the MEG efficiency is very sensitive to the condition of the NC surface and suggest that the wide range of MEG efficiencies reported in the recent literature may be a result of uncontrolled differences in NC surface chemistry.
\end{abstract}

Multiple exciton generation (MEG) in semiconductor nanocrystals (NCs) (also called quantum dots (QDs)) can produce $n$ excitons for each absorbed photon possessing an energy of at least $n$ multiples of the band gap energy $\left(E_{\mathrm{g}}\right)$, where $n$ is an integer. ${ }^{1-3}$ If multiexciton formation, dissociation, and charge collection are simultaneously efficient, the resulting enhanced photocurrent can increase solar energy conversion efficiencies. ${ }^{4,5}$ Recently, we reported a Schottky-junction photovoltaic device based on a thin film of colloidal $\mathrm{PbSe}$ NCs that demonstrated a power conversion efficiency of $>2 \%$ and a short-circuit current density, $J_{\mathrm{SC}}$, greater than $20 \mathrm{~mA} \mathrm{~cm}{ }^{-2}{ }^{6}$ The NC film in this device was treated with 1,2-ethanedithiol (EDT) in acetonitrile in a layer-by-layer procedure to produce a conductive $\mathrm{NC}$ film. ${ }^{7}$ Excitons are

\footnotetext{
*Corresponding authors: matt_beard@nrel.gov, anozik@nrel.gov.

† These authors contributed equally to this work.

* Basic Sciences Center, National Renewable Energy Laboratory.

${ }^{\S}$ Department of Chemistry and Biochemistry, University of Colorado.

"Present address: University of California, Irvine, CA.

${ }^{\perp}$ Department of Physics, University of Colorado.

" Present address: Department of Physics and Astronomy and Wright Center for Photovoltaics Innovation and Commercialization (PVIC), The University of Toledo.
}

created, separated, and transported all within the singlecomponent NC film. The internal quantum efficiency (IQE), defined as the fraction of photons absorbed by the NCs that produce carriers in the external circuit, was found to be as high as 0.8 , indicating efficient charge separation and transport. ${ }^{8}$ However, no evidence was found in the IQE spectra to suggest that multiple charge carriers were collected per absorbed photon. To harvest MEG excitons from a NC film, the inter-NC charge transfer event that produces free electrons and holes must be faster than Auger recombination (nonradiative exciton annihilation), which typically occurs in $10-100$ ps. In addition, the chemical treatments that are used to produce the conductive films must not degrade the MEG efficiency. We therefore conclude either that the EDT treatment quenches the MEG process or that multiexciton recombination is faster than inter-NC charge transfer (requiring exciton dissociation and charge separation).

In a previous report, we found that soaking $\mathrm{PbSe} \mathrm{NC}$ films in a solution of $1 \mathrm{M}$ hydrazine (hy) in acetonitrile $\left(\mathrm{CH}_{3} \mathrm{CN}\right)$ produces conductive films without degrading MEG. ${ }^{9}$ Here, we extend that work to the EDT $\mathrm{CH}_{3} \mathrm{CN}$ treatment and three other chemical treatments that produce conductive $\mathrm{PbSe} \mathrm{NC}$ 
solids: hy in ethanol (EtOH), methylamine (me) in EtOH, and pure EtOH. ${ }^{10}$ We show by ultrafast transient bleaching that EDT treatments almost completely quench MEG compared to pristine NC films prepared without any chemical treatment. Quenching of the MEG process by EDT explains why our previous device measurements failed to show MEGenhanced photocurrent. Finally, we find that the exciton quantum yield (QY) reproducibly increases when films of $3.7 \mathrm{~nm}$ diameter NCs are treated in pure $\mathrm{EtOH}$, while films with $7.4 \mathrm{~nm}$ NCs do not show such an increase.

We measure MEG by acquiring fast transients indicative of multiexciton states-which are short-lived compared to single exciton states - and relating the magnitude of the fast component relative to the long-lived component to the number of multiexcitons in the system. ${ }^{2,11}$ Transient absorption (TA) spectroscopy is a convenient technique with which to measure exciton population dynamics. Other time-resolved spectroscopies have also been employed to study MEG, including time-resolved photoluminescence (TRPL) ${ }^{12,13}$ and time-resolved terahertz spectroscopy (TRTS) ${ }^{14}$ which measures the intraband photoinduced absorption. ${ }^{15}$ These experimental probes provide complementary information. In general, TA provides a better measure of the carrier population dynamics than does TRPL because of its less restrictive selection rules.

Recent reports in the literature disagree as to the efficiency of MEG in several NC systems. For example, one study employing TA on InAs/CdSe/ZnSe core/shell/shell NCs did not find appreciable MEG, ${ }^{16}$ despite previous results from some of the same authors demonstrating very efficient MEG in this system using three techniques (TA, TRTS, and a quasi-cw PL technique).${ }^{14}$ Later the same group reported that their original TRTS results could not be reproduced on a new set of similarly prepared samples,${ }^{17}$ without mentioning whether the TA or the quasi-cw results also could not be reproduced. This apparent retraction and the later independent TA study are in contradiction to a TA study from Schaller and Kilmov ${ }^{18}$ showing enhanced QYs in InAs/ZnSe core/ shell NCs, but with a lower efficiency than found in the original Pijpers report. ${ }^{14}$ Similarly, using TRPL, Nair et al. ${ }^{12}$ are unable to reproduce the large quantum yields in $\mathrm{CdSe}$ NCs reported by Schaller and Klimov. ${ }^{13}$ Recently Nair et al. measured enhanced QYs in PbSe and PbS NCs employing TRPL ${ }^{19}$ but reported that MEG occurs at a lower efficiency than reported previously. ${ }^{2,3,20}$

These disagreements have cast doubt on the universality of MEG in NCs and suggest that sample-dependent phenomena may be responsible. It is conceivable that the surfaces of the NCs, which are not well characterized, controlled, or understood, can modulate the efficiency of the MEG process. It is known that surfaces of NCs have a large impact on their photophysical properties. Carrier relaxation $^{21,22}$ and carrier dynamics ${ }^{23}$ are affected by the surface ligands. Surface chemistry also determines the electrical properties of $\mathrm{NC}$ films, dictating in part the carrier doping level, mobility, and whether the films exhibit n- or p-type transport. ${ }^{7,10,23,24}$ To date no generally accepted theory has emerged to explain all of the MEG experimental findings. ${ }^{5}$
Since surface preparation can drastically modulate the carrier cooling rates, it is reasonable to conclude that MEG may also depend on the detailed chemistry and interactions at the NC surfaces. In the Shabaev-Efros-Nozik (SEN) MEG model, ${ }^{25}$ enhanced QYs are determined by the relative dephasing rates of single and multiexciton states that mix and form a coherent superposition of states through the multielectron Coulomb interaction. While measuring the dephasing rate of multiexciton states is experimentally challenging, the dephasing rate of single exciton states has been shown to depend upon the surface ligands. ${ }^{26}$ If dephasing rates depend on surface interactions, then MEG should also depend on surface interactions in the SEN model.

To date, no report has shown an absence of MEG in lead salt NCs; in fact a recent report from a group independent of those previously reporting on MEG confirms MEG in $\mathrm{PbSe} \mathrm{NCs} .{ }^{20} \mathrm{We}$ found a large variation in the enhanced QYs in our original paper on $\mathrm{PbSe}$ and $\mathrm{PbS} \mathrm{NCs.}{ }^{3}$ Understanding the variations in QYs and reconciling the different InAs, $\mathrm{PbSe}, \mathrm{PbS}$, and $\mathrm{CdSe} \mathrm{NC}$ results will enable a deeper understanding of the fundamental MEG process and may provide prospects for increased solar energy conversion efficiencies. Ben Lulu et al. ${ }^{16}$ rule out differences in experimental methods as the source of the different QYs for InAs-core NCs and suggest that the different $\mathrm{NC}$ structures - a core $/$ shell $_{1} /$ shell $_{2}$ versus just a core/shell-might explain the lack of measurable MEG with respect to the results of Schaller et al. ${ }^{18}$ While these authors consider sample-tosample variations as a possible contributor in their irreproducible results, they do not think this plays a significant role. In a recent report, ${ }^{27}$ McGuire et al. propose that uncontrolled, long-lived NC charging due to a low probability $\mathrm{NC}$ photoionization event may be the cause of the variations in the reported MEG efficiencies due to an accumulation of charged NCs over multiple laser shots. However, even when eliminating these extraneous effects by vigorously stirring of their samples, they report QYs higher than that found by Nair et al. ${ }^{19}$ However, a careful TA study by Trinh et al. ${ }^{20}$ did not find evidence for $\mathrm{NC}$ charging. In this report we have focused on NC films and show that very different MEG efficiencies are measured depending on how the NC films are prepared.

Results. We measured the multiexciton dynamics of two series of PbSe NC films, consisting of either 3.7 or $7.4 \mathrm{~nm}$ diameter NCs (first exciton transitions at 1310 and $2040 \mathrm{~nm}$ in tetrachloroethylene (TCE), respectively). The NCs were prepared according to published procedures, ${ }^{10}$ and films in each series were prepared from NCs produced in the same reaction. The NC films were spin cast directly onto the sapphire windows of $1.33 \mathrm{in}$. ConFlat viewports from 350 $\mathrm{mg} \mathrm{mL}{ }^{-1}$ octane solutions in a nitrogen-filled glovebox. Each film was then immersed in one of the following solutions for the following time: $0.02 \mathrm{M}$ EDT in $\mathrm{CH}_{3} \mathrm{CN}$ for $3 \mathrm{~min}$, or overnight in $1 \mathrm{M}$ hy in $\mathrm{CH}_{3} \mathrm{CN}, 1 \mathrm{M}$ hy in $\mathrm{EtOH}, 1 \mathrm{M}$ me in $\mathrm{EtOH}$, or pure $\mathrm{EtOH}$. After treatment, the films were allowed to dry in the glovebox and then sealed together with a second, uncoated ConFlat viewport to create an airtight sample cell that can remain outside of the glovebox for 


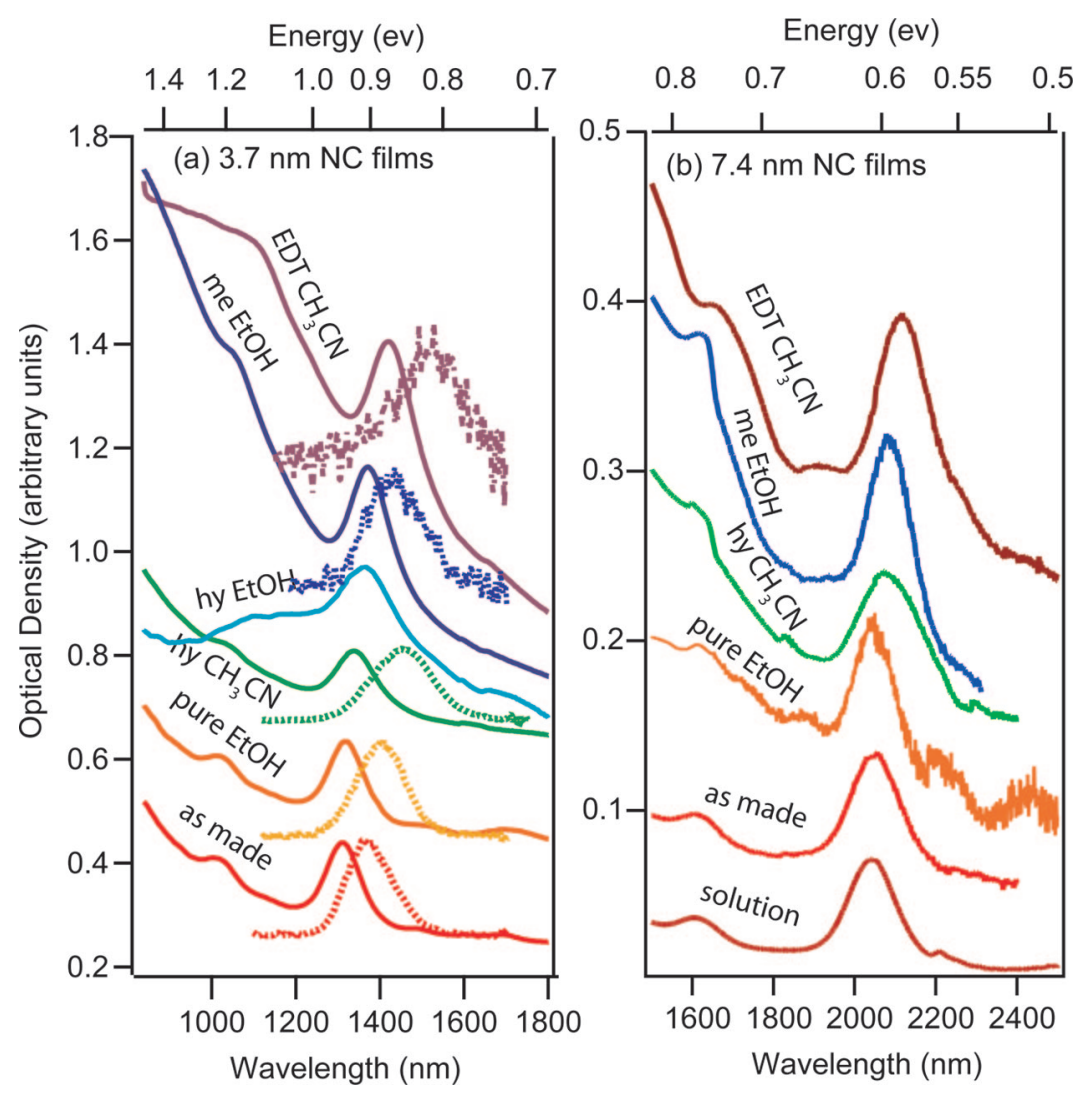

Figure 1. Extinction spectra of PbSe NC films, made from (a) $3.7 \mathrm{~nm}$ NCs (first exciton transition in TCE of $1310 \mathrm{~nm}$ ) and (b) $7.4 \mathrm{~nm}$ NCs (first exciton transition in TCE of $2040 \mathrm{~nm}$ ). Traces are offset for clarity. In (a) we also display the PL of the films. The PL is not reported for the large-sized NC.

several weeks without measurable change in the optical properties of the treated films. The basic characterization of these films is described in detail elsewhere. ${ }^{7,10}$ The size of the NCs is not affected by these chemical treatments. ${ }^{10}$ By investigating MEG for this series of film treatments, we hope to gain a better understanding of the overall factors that can enhance or reduce MEG.

Figure 1 shows the optical density near the first exciton transition for the 3.7 and $7.4 \mathrm{~nm} \mathrm{NC} \mathrm{films} \mathrm{(the} \mathrm{traces} \mathrm{have}$ been offset for clarity). While not shown here, the treated films experience an offset in their optical extinction due to scattering by cracking induced by loss of the oleate capping ligand during film treatment. Light scattering is also responsible for the negative slope on the red side of the first exciton transition. We consistently observe a red shift of the first exciton transition after chemical treatment due to a combination of changes in dielectric screening and inter-NC electronic coupling ${ }^{7,10}$ and a reduction in optical extinction at high photon energies relative to low photon energies, which we mainly attribute to a reduced dielectric screening as the discontinuity of the dielectric constant at the surface of the $\mathrm{NC}, \varepsilon_{\text {in }} / \varepsilon_{\text {out }}$, is reduced. ${ }^{7}$ The photoluminescence (PL) spectra of the $3.7 \mathrm{~nm} \mathrm{NC}$ films are displayed in Figure 1a, but we did not measure the PL from the $7.4 \mathrm{~nm} \mathrm{NC}$ films due to detector limitations. The photoluminescence quantum yields (PLQYs) of the $3.7 \mathrm{~nm}$ NC films are substantially lower than those of the NCs dispersed in TCE, with the PLQY roughly inversely proportional to the inter-NC separation previously determined by small-angle X-ray scattering. ${ }^{10}$ We display the PL normalized to the peak of the first exciton absorbance and do not attempt to quantify the absolute values of the film PLQYs. PL could not be detected from the hy EtOH treated films.

To measure MEG in the $3.7 \mathrm{~nm}$ NC films, we study the intensity-dependent interband TA bleach decays upon photoexciting with either 800 or $330 \mathrm{~nm}$ light, which corresponds to $\sim 1.6 E_{\mathrm{g}}$ and $\sim 4 E_{\mathrm{g}}$. For the $7.4 \mathrm{~nm} \mathrm{NC} \mathrm{films,} \mathrm{we} \mathrm{measured}$ the transient bleaching after photoexciting at 1300 and 400 $\mathrm{nm}$, which corresponds to $\sim 1.6 E_{\mathrm{g}}$ and $\sim 5 E_{\mathrm{g}}$. In all cases, the band edge bleach is monitored at the peak of the first exciton absorption. The experimental apparatus is described in detail elsewhere. ${ }^{5,9}$ Experiments with excitation light below that of the MEG threshold $\left(\sim 2 E_{\mathrm{g}}\right.$ ) allow us to obtain the single and biexciton lifetimes and also the absorption cross section, $\sigma_{\mathrm{a}}$. An exact knowledge of $\sigma_{\mathrm{a}}$ is not necessary to determine MEG. This is a critical feature of the data analysis because in the films the optical extinction may not be linearly related to $\sigma_{\mathrm{a}}$ at all wavelengths, due to wavelength-dependent scattering. To circumvent this complication, we measure the dependence of the carrier dynamics on pump fluences which, as we have shown previously, ${ }^{5,9,11}$ enables analysis that is insensitive to the exact knowledge of $\sigma_{\mathrm{a}}$.

The intensity-dependent carrier dynamics for photoexcitation of the $3.7 \mathrm{~nm}$ PbSe NC films at $800 \mathrm{~nm}$ are shown in Figure 2. Figure 3 displays the transients for the $7.4 \mathrm{~nm} \mathrm{NC}$ films with excitation light at $1300 \mathrm{~nm}$. We maintain a low excitation density so that the average exciton density per NC, $\left\langle N_{\mathrm{o}}\right\rangle$ at the front of the sample for the various incident photon 

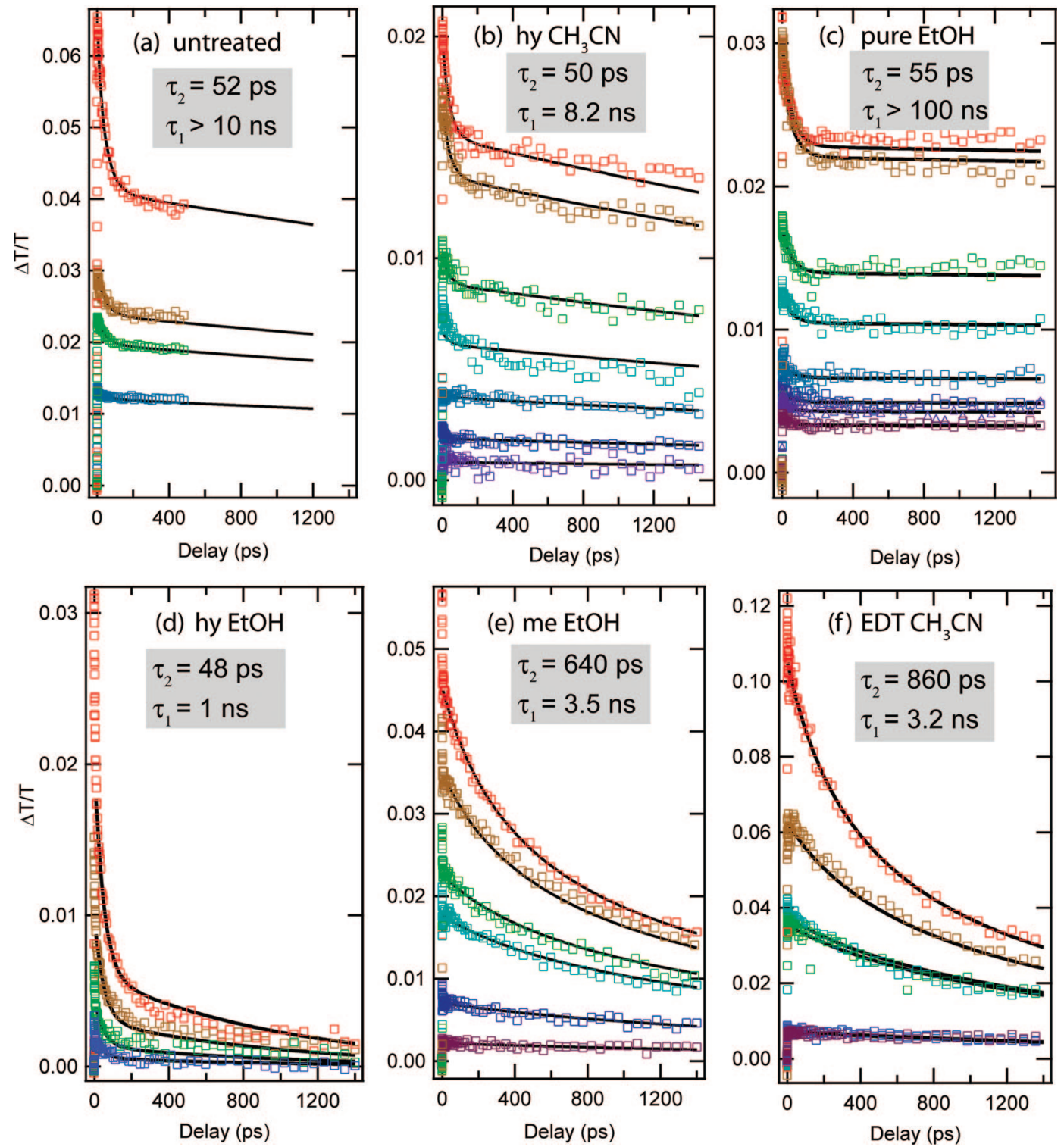

Figure 2. Intensity-dependent band edge bleach dynamics for $3.7 \mathrm{~nm} \mathrm{NC}$ films. The films were photoexcited at $\lambda_{\text {pump }}=800 \mathrm{~nm}$ which is $<2 E_{\mathrm{g}}$. The open squares are the data points, and the lines are the global fit described in the text. All data sets are modeled with the Auger recombination model discussed in the text except for the hy EtOH. The biexciton lifetime for these NCs in solution is $\sim 45 \mathrm{ps}$. For each film we report the average number of excitons per $\mathrm{NC}$ excited, $\left\langle N_{\mathrm{o}}\right\rangle$. (a) untreated, $\left\langle N_{\mathrm{o}}\right\rangle=0.22,0.39,0.49,1.09 ;(\mathrm{b}) \mathrm{hy} \mathrm{CH}_{3} \mathrm{CN},\left\langle N_{\mathrm{o}}\right\rangle=$ 0.022, 0.052, 0.11, 0.18, 0.28, 0.47, 0.54; (c) pure EtOH, $\left\langle N_{\mathrm{o}}\right\rangle=0.046,0.06,0.068,0.093,0.15,0.207,0.35,0.36$; (d) hy EtOH, fits to a biexponential function whose shape is independent of excitation fluence; (e) me EtOH, $\left\langle N_{\mathrm{o}}\right\rangle=0.088,0.287,0.72,0.92,1.42,1.84$; (f) EDT $\mathrm{CH}_{3} \mathrm{CN},\left\langle N_{\mathrm{o}}\right\rangle=0.185,0.188,0.19,0.92,0.98,1.63,2.76$.

fluences is low, ranging from $<0.02$ to $<2$ in Figures 2 and $3)$. This ensures that the average number of excitons per $\mathrm{NC}$ within the excitation volume is also low for shorter wavelengths with higher optical densities. Higher excitation intensities were found to gradually and irreversibly alter the decay dynamics over the course of the experiment, indicating photodamage of the samples. To avoid such damage, each transient was averaged over at least two traces. If scan-toscan reproducibility was not satisfactory, the resulting traces were not used in the analysis and another spot on the film was tested at a lower pump fluence. Using low photon densities also ensured that nonlinearities in $\Delta T / T$ did not complicate our analyses. All of the data were reproduced several times on each film and on at least two different films prepared at different times.
Each set of data is modeled using a global fitting routine that incorporates Auger recombination (AR) and is described in detail elsewhere. ${ }^{3,5}$ Within the global fitting routine, the single exciton lifetime $\left(\tau_{1}\right)$ and biexciton lifetime $\left(\tau_{2}\right)$ are varied globally across the entire data set; the best fit values are tabulated in Tables 1 and 2 and noted in Figures 2 and 3. There are three important changes that occur for the treated films relative to NCs dispersed in solution: (1) The single-exciton lifetime decreases for each of the chemical treatments except pure EtOH, reflecting an increased rate of surface recombination. (2) The biexciton lifetime increases as the inter-NC distance decreases. (3) The apparent absorption cross section per $\mathrm{NC}$ increases for films with longer biexciton lifetimes. These observations are consistent with our previous findings for drop-cast hy $\mathrm{CH}_{3} \mathrm{CN}$ treated films. ${ }^{9}$ 

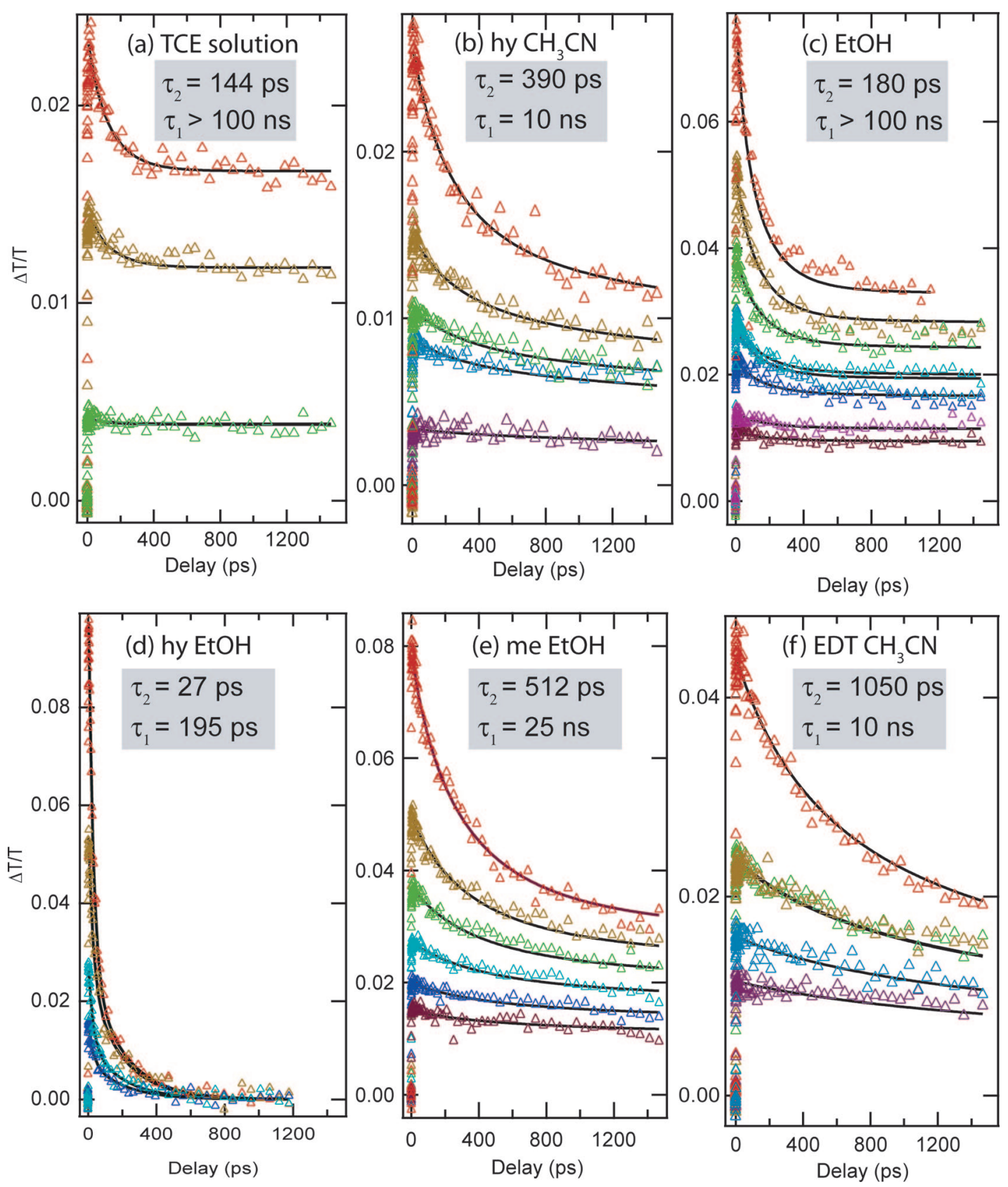

Figure 3. Intensity-dependent dynamics of $7.4 \mathrm{~nm} \mathrm{NC}$ films, $\lambda_{\text {pump }}=1300 \mathrm{~nm}$ : (a) solution of NCs in TCE, $\left\langle N_{\mathrm{o}}\right\rangle=0.13,0.46,0.74$; (b) hy $\mathrm{CH}_{3} \mathrm{CN},\left\langle N_{\mathrm{o}}\right\rangle=0.195,0.51,0.61,0.87,1.5$; (c) EtOH, $\left\langle N_{\mathrm{o}}\right\rangle=0.25,0.41,0.54,0.74,1.0,2.1$; (d) hy EtOH, the dynamics are characterized by a 195 ps time constant and a faster $27 \mathrm{ps}$ component at high photon fluences; (e) me EtOH, $\left\langle N_{\mathrm{o}}\right\rangle=0.29,0.58,0.71,1.0,1.4,2.0 ;$ (f) EDT $\mathrm{CH}_{3} \mathrm{CN},\left\langle N_{\mathrm{o}}\right\rangle=0.54,0.75,1.1,2.1$.

Table 1. Compilation of Results for $3.7 \mathrm{~nm} \mathrm{NC} \mathrm{Films}{ }^{a}$

\begin{tabular}{|c|c|c|c|c|c|c|c|}
\hline treatment & $\%$ oleate removed & $\sigma\left(\Omega^{-1} \mathrm{~cm}^{-1}\right)$ & $\Delta d(\mathrm{~nm})^{b}$ & type & $\tau_{1}$ & $\tau_{2}$ & $\mathrm{QY}$ \\
\hline solution & & & & & $>10 \mathrm{~ns}$ & $45 \mathrm{ps}$ & $1.5( \pm 0.1)$ \\
\hline untreated & & $<1 \times 10^{-9}$ & 0.0 & & $>10 \mathrm{~ns}$ & $52 \mathrm{ps}$ & $1.5( \pm 0.1)$ \\
\hline hy $\mathrm{CH}_{3} \mathrm{CN}$ & $60-70$ & $6 \times 10^{-3}$ & 0.8 & $\mathrm{n}$ & $8.2 \mathrm{~ns}$ & $50 \mathrm{ps}$ & $1.4( \pm 0.1)$ \\
\hline me EtOH & $80-85$ & $1 \times 10^{-5}$ & 0.9 & p & $3.5 \mathrm{~ns}$ & $640 \mathrm{ps}$ & $1.5( \pm 0.1)$ \\
\hline EtOH & $40-50$ & $2 \times 10^{-5}$ & 0.55 & $\mathrm{p}$ & $>100 \mathrm{~ns}$ & $55 \mathrm{ps}$ & $2.4( \pm 0.2)$ \\
\hline $\mathrm{EDT} \mathrm{CH}_{3} \mathrm{CN}$ & $99-100$ & $7 \times 10^{-4}$ & 1.8 & $\mathrm{p}$ & $3.2 \mathrm{~ns}$ & $862 \mathrm{ps}$ & $1.1( \pm 0.1)$ \\
\hline
\end{tabular}

${ }^{a}$ The average inter-NC distance in the untreated film is $\sim 1.8 \mathrm{~nm} .{ }^{b} \Delta d(\mathrm{~nm})$ is the change in inter-NC distance and represents the NCs moving closer together due to loss of the capping ligand, oleate. These results were obtained from Luther et al. ${ }^{7}$ and Law et al. ${ }^{10}$

The hy EtOH treated films show drastically shorter single-exciton lifetimes than the other treated films. The $3.7 \mathrm{~nm}$ NC films show biexponential decay with 48 and
1000 ps components, but neither component shows the variation with intensity indicative of biexciton decay. The first exciton lifetime decreased to $\sim 195$ ps for the $7.4 \mathrm{~nm}$ 
Table 2. Compilation of Results for the $7.4 \mathrm{~nm}$ NC Films

\begin{tabular}{|c|c|c|c|c|c|c|c|}
\hline treatment & $\%$ oleate removed & $\sigma\left(\Omega^{-1} \mathrm{~cm}^{-1}\right)$ & $\Delta d(\mathrm{~nm})^{a}$ & type & $\tau_{1}$ & $\tau_{2}$ & QY \\
\hline solution & & & & & $>100 \mathrm{~ns}$ & $144 \mathrm{ps}$ & $2.2( \pm 0.1)$ \\
\hline untreated & & $<1 \times 10^{-9}$ & 0.0 & & $5 \mathrm{~ns}$ & $150 \mathrm{ps}$ & $1.5( \pm 0.1)$ \\
\hline hy $\mathrm{CH}_{3} \mathrm{CN}$ & $30-35$ & $3 \times 10^{-2}$ & 0.8 & $\mathrm{n}$ & $10 \mathrm{~ns}$ & $390 \mathrm{ps}$ & $1.5( \pm 0.1)$ \\
\hline me EtOH & $80-85$ & $1 \times 10^{-4}$ & 0.9 & $\mathrm{p}$ & $25 \mathrm{~ns}$ & $512 \mathrm{ps}$ & $1.6( \pm 0.1)$ \\
\hline $\mathrm{EtOH}$ & $15-20$ & $3 \times 10^{-3}$ & 0.55 & $\mathrm{p}$ & $>100 \mathrm{~ns}$ & $180 \mathrm{ps}$ & $1.3( \pm 0.2)$ \\
\hline $\mathrm{EDT} \mathrm{CH}_{3} \mathrm{CN}$ & $99-100$ & $4 \times 10^{-3}$ & 1.8 & $\mathrm{p}$ & $10 \mathrm{~ns}$ & $1050 \mathrm{ps}$ & $1.0( \pm 0.1)$ \\
\hline
\end{tabular}

${ }^{a}$ These results were obtained from Luther et al. ${ }^{7}$ and Law et al. ${ }^{10}$
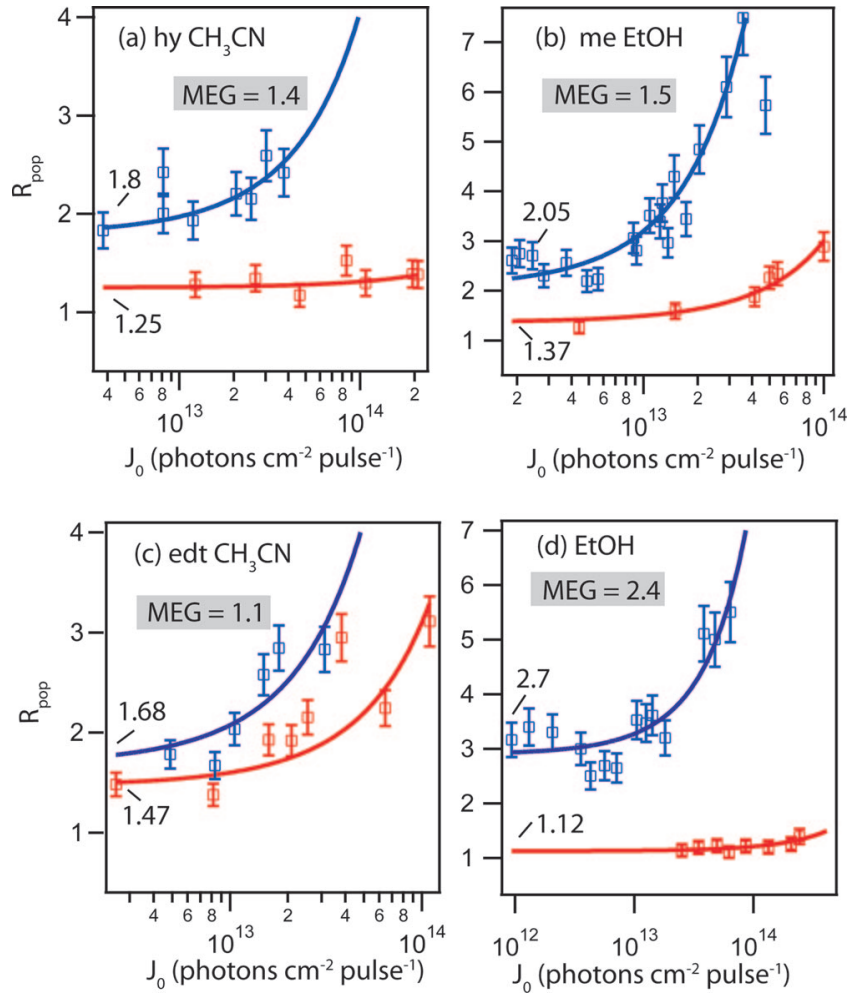

Figure 4. MEG results for the $3.7 \mathrm{~nm} \mathrm{NC}$ films: (a) hy $\mathrm{CH}_{3} \mathrm{CN}$; (b) me $\mathrm{EtOH}$; (c) $\mathrm{EDT} \mathrm{CH}_{3} \mathrm{CN}$; (d) $\mathrm{EtOH}$. Blue squares are for excitation at $330 \mathrm{~nm}\left(\sim 4 E_{\mathrm{g}}\right)$ and red squares are for excitation at $800 \mathrm{~nm}\left(\sim 1.6 E_{\mathrm{g}}\right)$. The solid lines are best fits of eq 1 .

NC films and a fast 27 ps component appears with higher pump fluence, indicative of a fast biexciton component. The 48 and 195 ps decays are roughly equal to that of the biexciton lifetimes found for the respective uncoupled NC films. As a result, we can tentatively assign this rapid decay to an Auger process involving a single exciton interacting with a charge carrier created by an ionized dopant. The hy EtOH treatment produces heavily n-type films (see below), with an estimated carrier density equivalent to $\sim 1$ electron per NC. The only other n-type films in the series are the hy $\mathrm{CH}_{3} \mathrm{CN}$ treated films, which apparently have a lower electron concentration and thus do not suffer from such pronounced Auger recombination. The short lifetimes of the hy EtOH films precluded measurements of their MEG efficiency by TA.

In Figures 4 and 5 we display the ratio, $R_{\text {pop }}$, of the TA signal at early times ( $2 \mathrm{ps})$ and late times (1500 ps). These

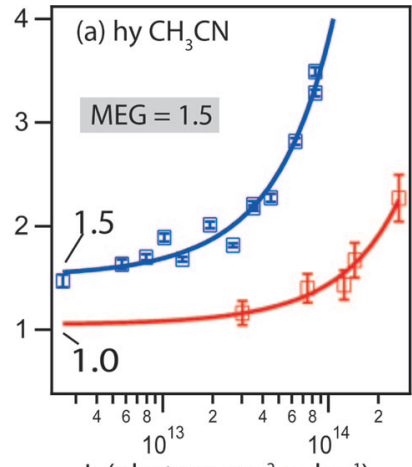

$\mathrm{J}_{0}$ (photons $\mathrm{cm}^{-2}$ pulse ${ }^{-1}$ )
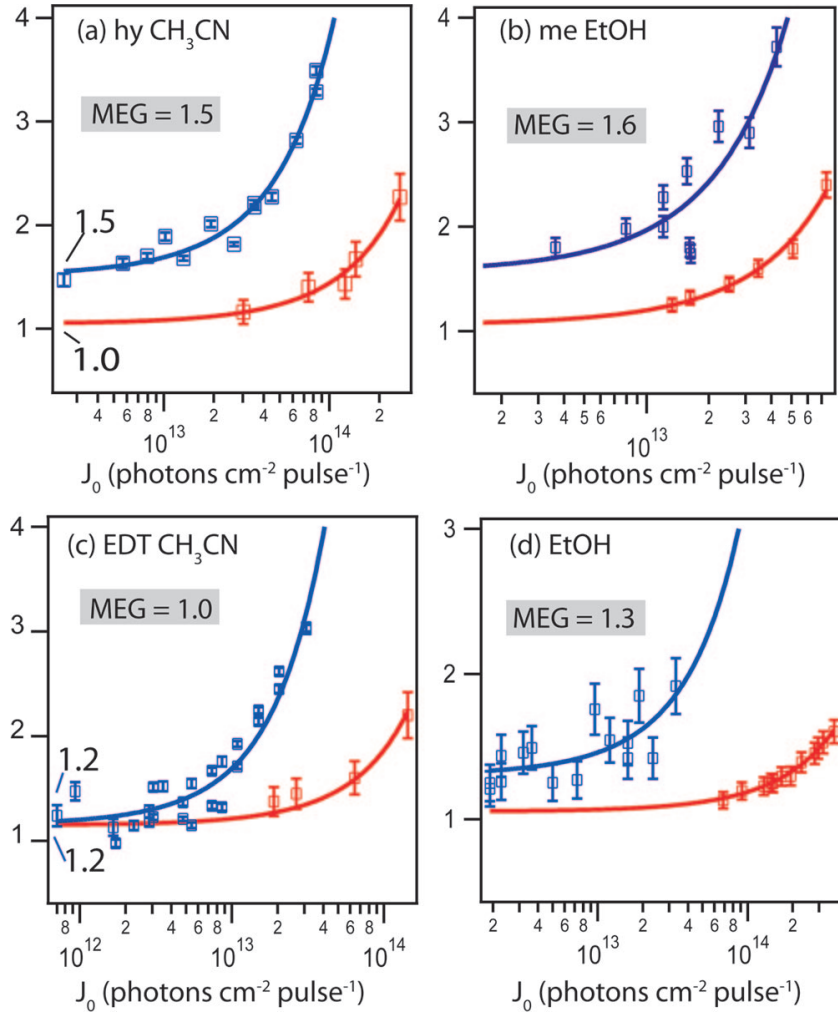

$\mathrm{J}_{0}$ (photons $\mathrm{cm}^{-2}$ pulse $\mathrm{e}^{-1}$ )

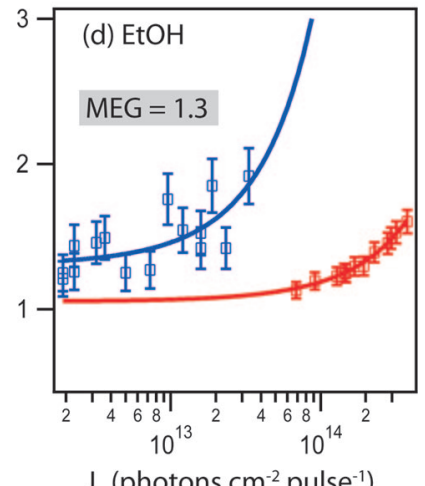

$\mathrm{J}_{0}$ (photons $\mathrm{cm}^{-2}$ pulse $\mathrm{s}^{-1}$ )

Figure 5. MEG results for the 7.4 NC films. Blue is for $400 \mathrm{~nm}$ excitation $\left(\sim 5 E_{\mathrm{g}}\right)$ and red is for $1300 \mathrm{~nm}$ excitation $\left(\sim 1.6 E_{\mathrm{g}}\right)$ : (a) hy $\mathrm{CH}_{3} \mathrm{CN}$; (b) me EtOH; (c) EDT $\mathrm{CH}_{3} \mathrm{CN}$; (d) $\mathrm{EtOH}$.

data are modeled with Poison statistics as previously described; $;{ }^{911}$ this analysis yields the following relationship

$$
R_{\text {pop }}=\frac{J_{0} \sigma_{\text {pump }} \delta \mathrm{QY}}{\left(1-\exp \left(-J_{0} \sigma_{\text {pump }}\right)\right)}
$$

where $J_{0}$ is the input photon fluence, $\sigma_{\text {pump }}$ is the absorption cross section per $\mathrm{NC}$ at the pump wavelength, and

$$
\delta=\exp \left\lfloor\left(t_{\text {late }}-t_{\text {early }}\right) / \tau_{1}\right\rfloor
$$

accounts for the decay of the single exciton. The $y$-intercept for the sub-MEG threshold excitation (red squares are the data points and the red line is the best fit values) is indicative of the decay of the first exciton and determines $\delta$. The ratio of the $y$-intercept for above-MEG threshold (blue squares are the data points and the best fit is the blue line) and subMEG threshold determines the QY; this is equivalent to holding $\delta$ fixed at the value determined by fitting the subMEG threshold data and floating QY in eq 1. For the hy $\mathrm{CH}_{3} \mathrm{CN}$ treated $3.7 \mathrm{~nm} \mathrm{NC}$ film, the QY is $1.8 / 1.25=1.4$ \pm 0.1 . The $3.7 \mathrm{~nm}$ NCs suspended in TCE, and the untreated film (data not shown) showed QYs of $\sim 1.5 \pm 0.1$. The me $\mathrm{CH}_{3} \mathrm{CN}$ and hy $\mathrm{CH}_{3} \mathrm{CN}$ treated $3.7 \mathrm{~nm} \mathrm{NC}$ films show QYs 


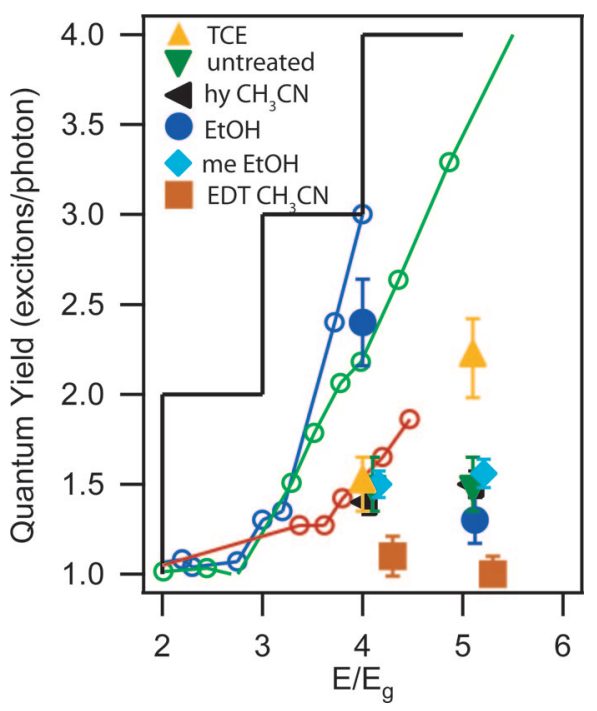

Figure 6. QYs as determined in this work (solid symbols) overlaid with past QYs of PbSe NCs dispersed in solution from NREL, ref 3. Blue open circles correspond to results for $4.0 \mathrm{~nm} \mathrm{NCs}$ in TCE and the red open circles are average results for 4.7 and $5.7 \mathrm{~nm}$ $\mathrm{NCs}$ in TCE. In our original report some variation on either sample preparation or NC size was observed. The green open circles are reported from LANL, ref 18 . The solid black line represents the maximum possible MEG efficiency; achieving $n$ excitons at $n$ times the band gap energy.

unchanged from the untreated NCs, in agreement with our previous findings. However, the EDT $\mathrm{CH}_{3} \mathrm{CN}$ and the pure EtOH $3.7 \mathrm{~nm}$ NC treated films show substantially different QYs of $1.1 \pm 0.1$ and $2.4 \pm 0.2$, respectively.

Figure 5 presents the MEG results for the $7.4 \mathrm{~nm} \mathrm{NC}$ films treated with hy $\mathrm{CH}_{3} \mathrm{CN}$, me EtOH, EDT $\mathrm{CH}_{3} \mathrm{CN}$, and EtOH: results for the NCs in TCE and the as-made films can be found in the Supporting Information. We find similar behavior to the $3.7 \mathrm{~nm} \mathrm{NC}$ films for the EDT $\mathrm{CH}_{3} \mathrm{CN}$ treatment: MEG is largely quenched. For the hy $\mathrm{CH}_{3} \mathrm{CN}$, me EtOH, and the untreated $7.4 \mathrm{~nm} \mathrm{NC}$ films we find that MEG is reduced compared to the NCs in solution, with QY $=1.5-1.6$ in the films compared to $2.2( \pm 0.1)$ for the isolated NCs. In contrast to the smaller NCs where EtOH treatment produced the highest MEG efficiency $(\mathrm{QY}=2.4)$, the EtOH-treated larger NCs showed a QY of $1.3( \pm 0.1)$, slightly reduced relative to the other films. In each case the results indicate a reduction in MEG for the larger NCs relative to the same film treatments for the smaller NCs. Figure 6 compiles the MEG results from this study with our previous results. The blue open circles are data taken for $4.0 \mathrm{~nm} \mathrm{NCs}$ and the red circles are for larger NCs, 4.7 and $5.7 \mathrm{~nm}$ diameters. The open green circles are data reproduced from Schaller et al. ${ }^{28}$ for isolated PbSe NCs.

The percentage of oleate removed after treatment from small and large NCs was quantified by an FTIR analysis (see Supporting Information) and is similar to that reported

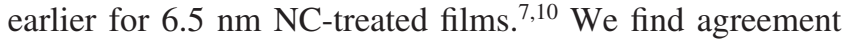
with our previous findings for the $\mathrm{EDT} \mathrm{CH}_{3} \mathrm{CN}$, me $\mathrm{EtOH}$, and hy EtOH treatments and no significant dependence on the NC size. However, we consistently find larger oleate loss for the hy $\mathrm{CH}_{3} \mathrm{CN}$ treatment, $30-35 \%$ in this study versus only $2-7 \%$ in our previous findings. We also find a size- dependent oleate loss for both the hy $\mathrm{CH}_{3} \mathrm{CN}$ and pure $\mathrm{EtOH}$ treatments. Thus, while the large NCs treated with $\mathrm{EtOH}$ show consistent results with our previous findings, the smaller NCs lose more oleate, $50 \%$ vs $15-20 \%$. The effect of hy $\mathrm{CH}_{3} \mathrm{CN}$ treatment is also size-dependent; the smaller NCs lose $60-70 \%$ while the larger NCs lose $30-35 \%$. In our earlier report the loss of oleate was correlated with a decrease in inter-NC separation, quantified by small-angle $\mathrm{X}$-ray scattering. We assume here that the trends in changes to the inter-NC separation are the same as those found in our earlier studies. We repeated our earlier electrical characterization to determine if film conductivity depends on NC size. We are able to reproduce the earlier results ${ }^{7,10}$ for all of the film treatments except $\mathrm{EDT} \mathrm{CH}_{3} \mathrm{CN}$, where we observe an order of magnitude higher conductivity (see Supporting Information for a comparison of our recent measurements with those reported previously). We consistently observe higher conductivities for the larger NCs compared to the smaller NCs; however, the trends in the conductivity with chemical treatment are size-independent. Table 1 and 2 summarize the MEG results, single and biexciton lifetimes, conductivities, and the change in inter$\mathrm{NC}$ separation for the small and large NCs, respectively.

Discussion. There are at least three ways in which the various chemical treatments can affect MEG: (1) changes in the surface chemistry which affect NC-ligand interactions and subsequently exciton relaxation rates; (2) doping of the NCs causing enhanced Auger recombination; (3) an increase in inter-NC coupling producing diminished quantum confinement and/or charge-transfer states with higher carrier mobilities and lower inter-NC charge-transfer barriers. While the present study cannot distinguish which of these is most important to MEG, we can gain insight by discussing the trends.

The variations in MEG are not correlated with the fraction of oleate removed from the NC films by the chemical treatments. For instance, the me EtOH treated films result in an 80-90\% loss of oleate while the hy $\mathrm{CH}_{3} \mathrm{CN}$ treatment results in 33\% oleate loss; however, MEG is about the same in the two films. Moreover, the $3.7 \mathrm{~nm}$ EtOH film loses $40-50 \%$ compared to only $15-20 \%$ for the larger NCs, while MEG is enhanced in the smaller NCs and reduced in the larger NCs. The me EtOH and hy EtOH treatments remove about the same amount of oleate and cause a similar reduction in the inter-NC spacing. However, the electrical properties and carrier dynamics are vastly different after these two treatments. The hy EtOH treatment produces n-type films with large conductivities and fast single-exciton decay while me EtOH produces p-type films with lower conductivities and only slightly reduced single-exciton decays. As the loss of oleate in itself is insufficient to determine either the electrical behavior of the films, the carrier dynamics, or MEG, we conclude that other factors such as inter-NC separation or the chemical reactivity of the treatments are more important.

The chemical treatments do more than simply remove oleate from the NCs. Of the four treatments, hydrazine is 
the strongest reducing agent. Treating the films in pure hy, or a concentration substantially higher than $1 \mathrm{M}$, yields measurable metallic $\mathrm{Pb}$ in the film. ${ }^{10}$ Hydrazine therefore tends to reduce surface $\mathrm{Pb}^{2+}$ to produce n-type films. While methylamine is a reducing agent, it is apparently unable to reduce the surface $\mathrm{Pb}^{2+}$ as efficiently as hydrazine. p-Type films may result if the as-produced NCs are p-type, which can be the case if the NCs are Se-rich. Treatments may also produce p-type films by partially oxidizing the NC surfaces. The identity of the NC surface species after chemical treatment, whether adsorbates, vacancies, or other surface states, is difficult to ascertain and quantify. Small amounts of impurities in the EtOH or other chemical treatments such as residual water may alter the inter-NC coupling through lowering of barrier heights for efficient transport and/or oxidizing or reducing surface $\mathrm{Pb}$ or $\mathrm{Se}$ ions. The precise nature of the resulting surface species may dictate much of the electrical and carrier dynamics observed here. A small defect concentration, corresponding to only 1 defect site in 10 to $100 \mathrm{NCs}$, could produce large changes in the measured conductivities. For the EDT $\mathrm{CH}_{3} \mathrm{CN}$ treated films producing p-type conductivity, an estimate of the carrier concentration has been obtained through Mott-Schottky analysis ${ }^{6}$ yielding carrier densities of $\sim 10^{16}$ to $10^{17} \mathrm{~cm}^{-3}$. These carrier densities correspond to approximately 1 dopant in 10-100 NCs, which agrees with an estimate of the carrier doping levels in similarly prepared hy $\mathrm{CH}_{3} \mathrm{CN}$-treated films. ${ }^{29}$ The conductivity of the hy EtOH treatments are an order of magnitude higher; therefore, the dopant density is likely higher, yielding values approaching 1 dopant per NC. Such low amounts of surface doping are very difficult to measure using standard techniques such as Fourier transform infrared (FTIR) spectroscopy and X-ray photoelectron spectroscopy (XPS). ${ }^{10} \mathrm{~A}$ $3.7 \mathrm{~nm} \mathrm{NC}$ has approximately 1000 surface atoms, requiring detection of 1 part in 1000. To highlight this point, we draw attention to measurements on hy $\mathrm{CH}_{3} \mathrm{CH}$ treated films that show no evidence for hy in the FTIR or XPS analysis after treatment. Upon heating, the film switches from n-type to p-type as the small amount of hy (or other absorbates) is desorbed from the film. n-Type conductivity is restored upon retreating the film with hy $\mathrm{CH}_{3} \mathrm{CN} .{ }^{10}$ The amount of hy that is desorbed and then readsorbed is smaller than our detection limits.

Carrier concentrations approaching 1 dopant per NC could result in new Auger recombination pathways for nonradiative recombination of single excitons and may explain the fast decays observed in the hy EtOH films as discussed previously. We do not see evidence for this mechanism in the hy $\mathrm{CH}_{3} \mathrm{CN}$ treated films which are also n-type and have similarly high conductivities. The reason for this difference is unknown but may suggest a different mechanism for the fast decays in the hy EtOH treated films. It is important to note here that charged NCs, if present, would cause a fast decay in the single-exciton dynamics that is photon-energy independent. MEG causes a fast decay in the apparent single-exciton dynamics that is photon-energy dependent; the fast component is larger at higher photon energies. The small amount of static charges (produced by doping) in the film does not

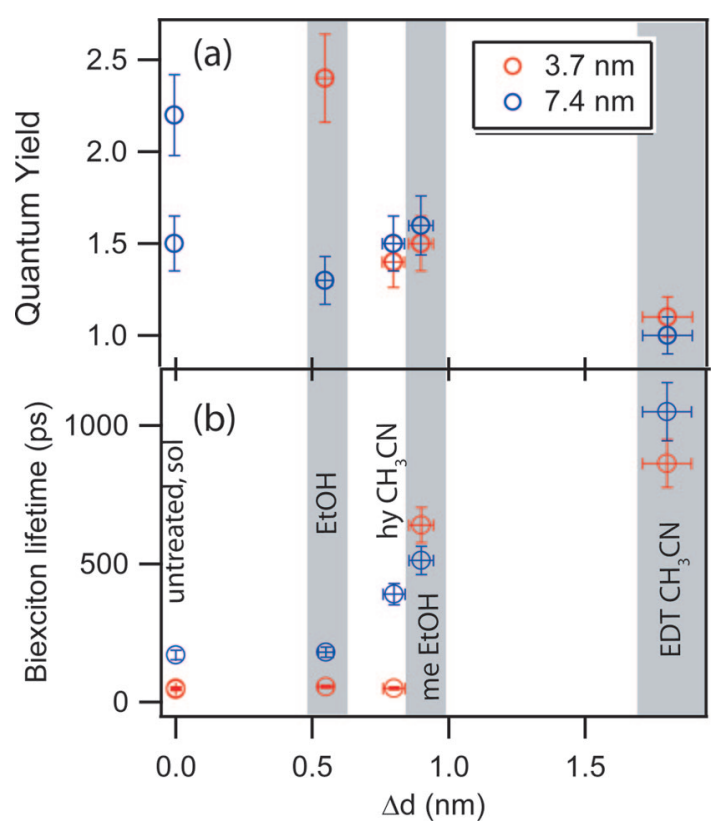

Figure 7. Correlation of QY (a) and biexciton lifetime (b) with the change in average inter-NC distance for the $3.7 \mathrm{~nm} \mathrm{NC}$ films (red circles) and $7.4 \mathrm{~nm} \mathrm{NC} \mathrm{films} \mathrm{(blue} \mathrm{circles).}$

appear to influence the optical data in any of the films except perhaps the hy EtOH film. Transient charging of the films due to photoionization appears not to play a significant role in these results. The MEG efficiency of all of the films except the EtOH-treated film decreased or remained the same as the NCs in solution. It is not apparent how transient charging of the NCs could account for an apparent reduction in MEG efficiency. It may be that low probability ionization events such as those proposed by McGuire et al. ${ }^{27}$ do not produce long-lived charged states in these films due to the high mobility of carriers.

While MEG is not correlated with loss of oleate, the MEG efficiency does correlate with the change in inter-NC distance for both NC sizes, with smaller NC separation coinciding with lower MEG yields. Figure 7a plots the QY versus the change in inter-NC distance created by the various film treatments. This correlation could arise due to an increase in inter-NC electronic coupling that bypasses or inhibits the MEG process. As neighboring NCs couple more strongly to one another, quantum confinement could be reduced sufficiently to greatly decrease the Coulomb coupling necessary to drive the MEG process. Furthermore, charge-transfer states, where the electron resides on one $\mathrm{NC}$ and the hole on an adjacent $\mathrm{NC}$, could become lower in energy than highly excited single exciton states isolated on single NCs, as an example see ref 30. In a model that treats MEG, excitation at higher photon energies would result in a mixture of chargetransfer states, single exciton states, and multiexciton states, and the interplay between these states could decrease MEG by rapid population of charge-transfer states. The final state of the system, and hence any enhanced QYs, would then be determined by the relative dephasing and carrier cooling rates within the different channels that include the charge-transfer states. Recently, we reported that the IQE of solar cells constructed from $\mathrm{PbSe} \mathrm{NC}$ films treated with EDT $\mathrm{CH}_{3} \mathrm{CN}$ 
decreases in the IR region of the spectrum, suggesting the existence of a branching ratio between excitonic states and charge-transfer states that depends on the photon energy. ${ }^{8}$ This observation suggests that the formation of chargetransfer states competes with exciton cooling which occurs on a picosecond time scale and therefore provides tentative evidence that charge-transfer states could also play a role in the decreased MEG efficiency. A similar situation arises in molecular solids of pentacene where charge-transfer states can be directly photoexcited despite weak intermolecular interactions. ${ }^{31}$ More work is needed to fully understand these observations and will be the topic of future reports.

In support of the above conclusions, the increase in biexciton lifetime, $\tau_{2}$, is also correlated to the change in interNC distance (see Figure 7b). The increase of $\tau_{2}$ with smaller inter-NC separation indicates a reduced Auger recombination rate. We attribute this to a delocalization of the excitons due to increased electronic coupling between NCs. The increased $\tau_{2}$ is greatest for the EDT $\mathrm{CH}_{3} \mathrm{CN}$ treated film, where $\tau_{2}$ increases by an order of magnitude relative to untreated films. The red shift in the first exciton and the decrease in the interNC spacing are also largest for the EDT-treated films. The me EtOH treated films also a show substantially increased $\tau_{2}$. Consistent with our previous findings, the biexciton lifetimes of the hy $\mathrm{CH}_{3} \mathrm{CN}$ treated films increase for the larger NCs, from 150 to $390 \mathrm{ps,} \mathrm{while} \mathrm{only} \mathrm{increasing} \mathrm{slightly}$ for the smaller NCs. ${ }^{9}$ We conclude that electronic coupling is strongest in the EDT $\mathrm{CH}_{3} \mathrm{CN}$, followed by me $\mathrm{EtOH}$, hy $\mathrm{CH}_{3} \mathrm{CN}$, and finally the EtOH films. This roughly correlates with the MEG efficiency, suggesting a tradeoff between MEG and strong electronic coupling accompanied by reduced quantum confinement.

The results for the EtOH treated films, while showing roughly the correlation with inter-NC distance, also demonstrate anomalous behavior. The MEG efficiency is greatly increased in the smaller NC films, while it decreases in the larger NC films. While the biexciton lifetime of the EtOH treated smaller NCs remains approximately the same as that for the untreated NCs, the single exciton lifetime increases, suggesting that the surfaces are better passivated. It is unclear why the EtOH treatment increases the surface passivation as a significant amount of oleate is removed. Some evidence of either absorbed molecular ethanol and/or ethoxide on the $\mathrm{NC}$ surfaces was found, ${ }^{10}$ and these species could account for the better passivation relative to oleate. The increased surface passivation may partially explain the increase in MEG observed in the smaller NCs. In contrast, the larger NCs lose less oleate with a concomitant lower MEG. The effect on the single-exciton lifetime of the $\mathrm{EtOH}$ on the larger NCs is hard to ascertain given the $1.2 \mathrm{~ns}$ time window used here. The fact that the EtOH treated films show anomalous behavior suggests that the issues governing MEG are still unresolved and require further study with careful control over doping and intimate knowledge of the NC surfaces.

In conclusion, we have measured MEG by transient absorption in a series of chemically treated conductive $\mathrm{PbSe}$ NC films. We observe a large variation in the MEG efficiency depending on the NC size and the chemical treatment. The decrease in MEG with smaller inter-NC distance may indicate that reduced quantum confinement and/ or an increased inter-NC coupling diminish the MEG process. This large sensitivity of the QYs to conditions at the NC surface suggests that recent disagreements over the magnitude of the QYs of various colloidal NC systems may stem from uncontrolled differences in NC surface chemistry. Our results, combined with recent demonstrations that solar cells with large photocurrents can be fabricated from $\mathrm{PbSe} \mathrm{NCs}$, ${ }^{6,32}$ provide an incentive to study the surfaces of NCs in detail, with the specific goal of understanding how different ligands and surface states affect carrier dynamics and electrical transport within NC films. A better understanding of how the NC surfaces modulate the electrical properties, carrier dynamics, and MEG efficiency is clearly desired in order to design systems that harness MEG to convert light into electricity with very high efficiency.

Acknowledgment. We thank Barbara Hughes and Qing Song for synthesis of the NCs. We acknowledge Hugh Hillhouse and Justin Johnson for helpful discussions. M.C.B., A.G.M., and A.J.N. gratefully acknowledge support from the Division of Chemical Sciences, Geosciences, and Biosciences, Office of Basic Energy Sciences, within the Department of Energy. M.L. was supported by the DOE EERE photovoltaics program and O.E.S. by a DARPA grant. DOE funding is provided by the Department of Energy, under Contract DE-AC36-08GO28308 to the National Renewable Energy Laboratory.

Supporting Information Available: Figures showing amount of oleate loss for each film, comparison of conductivity results for this study and previous results, and MEG for the $7.4 \mathrm{~nm}$ NCs. This material is available free of charge via the Internet at http://pubs.acs.org.

\section{References}

(1) Nozik, A. J. Physica E 2002, 14, 115.

(2) Schaller, R.; Klimov, V. Phys. Rev. Lett. 2004, 92, 186601.

(3) Ellingson, R. J.; Beard, M. C.; Johnson, J. C.; Yu, P.; Micic, O. I.; Nozik, A. J.; Shabaev, A.; Efros, A. L. Nano Lett. 2005, 5, 865.

(4) Hanna, M. C.; Nozik, A. J. J. Appl. Phys. 2006, 100, 074510/1.

(5) Beard, M. C.; Ellingson, R. J. Laser Photonics Rev. 2008, $2,377$.

(6) Luther, J. M.; Law, M.; Beard, M. C.; Song, Q.; Reese, M. O.; Ellingson, R. J.; Nozik, A. J. Nano Lett. 2008, 8, 3488.

(7) Luther, J. M.; Law, M.; Song, Q.; Perkins, C. L.; Beard, M. C.; Nozik, A. J. ACS Nano 2008, 2, 271.

(8) Law, M.; Beard, M. C.; Choi, S.; Luther, J. M.; Hanna, M.; Nozik, A. J. Nano Lett. 2008, 8, 3904.

(9) Luther, J. M.; Beard, M. C.; Song, Q.; Law, M.; Ellingson, R. J.; Nozik, A. J. Nano Lett. 2007, 7, 1779.

(10) Law, M.; Luther, J. M.; Song, O.; Hughes, B. K.; Perkins, C. L.; Nozik, A. J. J. Am. Chem. Soc. 2008, 130, 5974.

(11) Beard, M. C.; Knutsen, K. P.; Yu, P. R.; Luther, J. M.; Song, Q.; Metzger, W. K.; Ellingson, R. J.; Nozik, A. J. Nano Lett. 2007, 7, 2506.

(12) Nair, G.; Bawendi, M. G. Phys. Rev. B 2007, 76, 081304.

(13) Schaller, R. D.; Sykora, M.; Jeong, S.; Klimov, V. I. J. Phys. Chem. B 2006, 110, 25332.

(14) Pijpers, J. J. H.; Hendry, E.; Milder, M. T. W.; Fanciulli, R.; Savolainen, J.; Herek, J. L.; Vanmaekelbergh, D.; Ruhman, S.; Mocatta, D.; Oron, D.; Aharoni, A.; Banin, U.; Bonn, M. J. Phys. Chem. C 2007, 111, 4146.

(15) Beard, M. C.; Turner, G. M.; Schmuttenmaer, C. A. Nano Lett. 2002 , 2, 983.

(16) Ben-Lulu, M.; Mocatta, D.; Bonn, M.; Banin, U.; Ruhman, S. Nano Lett. 2008, 8, 1207. 
(17) Pijpers, J. J. H.; Hendry, E.; Milder, M. T. W.; Fanciulli, R.; Savolainen, J.; Herek, J. L.; Vanmaekelbergh, D.; Ruhman, S.; Mocatta, D.; Oron, D.; Aharoni, A.; Banin, U.; Bonn, M. J. Phys. Chem. C 2008, 112, 4783.

(18) Schaller, R. D.; Pietryga, J. M.; Klimov, V. I. Nano Lett. 2007, 7, 3469.

(19) Nair, G.; Geyer, S. M.; Chang, L. Y.; Bawendi, M. G. Phys. Rev. B 2008, 78, 10 .

(20) Trinh, M. T.; Houtepen, A. J.; Schins, J. M.; Hanrath, T.; Piris, J.; Knulst, W.; Goossens, A.; Siebbeles, L. D. A. Nano Lett. 2008, 8, 1713.

(21) Guyot-Sionnest, P.; Wehrenberg, B.; Yu, D. J. Chem. Phys. 2005, 123, 074709.

(22) Pandey, A.; Guyot-Sionnest, P. Science 2008, 322, 929.

(23) Murphy, J. E.; Beard, M. C.; Nozik, A. J. J. Phys. Chem. B 2006, 110,25455 .

(24) Talapin, D. V.; Murray, C. B. Science 2005, 310, 86.
(25) Shabaev, A.; Efros, A. L.; Nozik, A. J. Nano Lett. 2006, 6, 2856.

(26) Salvador, M. R.; Hines, M. A.; Scholes, G. D. J. Chem. Phys. 2003, $118,9380$.

(27) McGuire, J. A.; Joo, J.; Pietryga, J. M.; Schaller, R. D.; Klimov, V. I. Acc. Chem. Res. 2008, 41, 1810.

(28) Schaller, R. D.; Sykora, M.; Pietryga, J. M.; Klimov, V. I. Nano Lett. 2006, 6, 424.

(29) Wang, R. Y.; Feser, J. P.; Lee, J. S.; Talapin, D. V.; Segalman, R.; Majumdar, A. Nano Lett. 2008, 8, 2283.

(30) Scholes, G. D. ACS Nano 2008, 2, 523.

(31) Schuster, R.; Knupfer, M.; Berger, H. Phys. Rev. Lett. 2007, 98, 4.

(32) Johnston, K. W.; Pattantyus-Abraham, A. G.; Clifford, J. P.; Myrskog, S. H.; MacNeil, D. D.; Levina, L.; Sargent, E. H. Appl. Phys. Lett. 2008, 92, 3.

NL803600V 